\title{
Standard and modified techniques for parenchyma-preserving hepatectomy focused on segments I + IV resection in patients with perihilar cholangiocarcinoma
}

\author{
Shin HWANG*
}

Department of Surgery, Asan Medical Center, University of Ulsan College of Medicine, Seoul, Korea

Introduction: Resection of the hepatic segments I + IV $(\mathrm{S} 1+\mathrm{S} 4)$ is the most common type of parenchyma-preserving hepatectomy (PPH) for perihilar cholangiocarcinoma (PHCC).

Methods: We describe our experience on the standard and modified techniques for PPH focused on S1 + S4 resection in patients with PHCC.

Results: 1) Isolated caudate lobectomy with bile duct resection (BDR) is the minimal type of PPH, but not currently recommended due to technical difficulty. 2) Partial hepatectomy of S1 + S4a \pm segment V (S5) with BDR provides wide operative field, but extension of BDR is limited and resection of S1 paracaval portion is still difficult. 3) Resection of S1 + S4 + S5 with BDR provides wider operative field for complete S1 resection and multiple biliary reconstruction. 4) Resection of S1 + S4 with BDR offers very wide operative field and allows wider extent of hilar BDR, and thus presents the most common type of PPH. A supplementary video clip presents the detailed standard surgical procedure for resection of S1 + S4 with BDR in a patient with type IIIA PHCC. 5) Modified resection of S1 + S4 \pm S5 or segment VIII (S8) with BDR facilitates additional resection of tumor-involved S5 or S8 ducts. 6) Major hilar vascular invasion is usually contraindicated for PPH and only small portal vein invasion requiring wedge resection and patch venoplasty is allowed.

Conclusions: In conclusion, PPH can achieve curative resection and improved outcomes in patients with PHCC via reasonable modification of the extent of hepatectomy and hilar BDR. PPH may have advantages in selected patients depending on the extent of tumor, and in patients with high operative risk. 